\title{
Application Using Standard Communication Between Medical Facilities
}

\author{
Mihaela CRISAN-VIDA ${ }^{\mathrm{a}, 1}$, Iulia GOLEA ${ }^{\mathrm{a}}$, Răzvan BOGDAN ${ }^{\mathrm{a}}$ and \\ Lăcrămioara STOICU-TIVADAR ${ }^{\mathrm{a}}$ \\ ${ }^{a}$ Faculty of Automation and Computers, University Politehnica Timisoara, Romania
}

\begin{abstract}
The web-based application described in this paper will support the patient to receive the treatment quicker and the physician to generate the prescription easier. The patient will have a real time information if their treatment/prescription is available in the pharmacy. Using a cloud solution will have all the information always available and without delays, the only requirement is the Internet connectivity. Using standardized communication as HL7 FHIR, the information exchanged is easier understanded by different medical units, and in the future other medical units can access the patient treatment/prescription and have a medical history, in this way the patient will receive better quality in treatment and health care.
\end{abstract}

Keywords. Web-based application, cloud computing, interoperability, HL7 FHIR

\section{Introduction}

In the current healthcare context one of the important things in digital health is interoperability. The interoperability in healthcare domain is defined as: "exchange of information between different entities and the received information to be understand by all the entities" [1]. This interoperability can be achieved by using a standardized communication, as HL7 FHIR (Fast Healthcare Interoperability). FHIR [2] is a standard for healthcare data exchange published by HL7 organization. FHIR specification describes a set of base resources, frameworks and APIs that can be use in different contexts in digital healthcare. For having the web-based application always available we provide a cloud computing solution, based in our case on Microsoft Azure [3] different services, hosting the web-based application and API for FHIR.

This paper presents the communication between the medical unit and pharmacies using HL7 FHIR. One of the main issues in Romania is that the medical unit generates a prescription with electronic support, but only for compensated or free prescription, and the physician gives it to the patient in paper format. The natural process isthe electronic support, where the medical entity sends in a standardized way the prescription to the pharmacy. After an analysis of applications on the market, as Dataklas Pharmaceutical [4], Pharmec [4], BizPharma [4] the conclusion was that they could not receive prescriptions in a standardized way.

${ }^{1}$ Corresponding Author, Mihaela Crișan-Vida, Faculty of Automation and Computers, University Politehnica Timisoara, Romania, Bd. Vasile Pârvan no. 2, Timisoara, Romania; E-mail: mihaela.vida@upt.ro. 


\section{Materials and methods}

Figure 1 presents the system architecture where a medical unit communicates with the pharmacy in a standardized way. The cloud computing facility is useful ensuring to have the communication and the information always available.

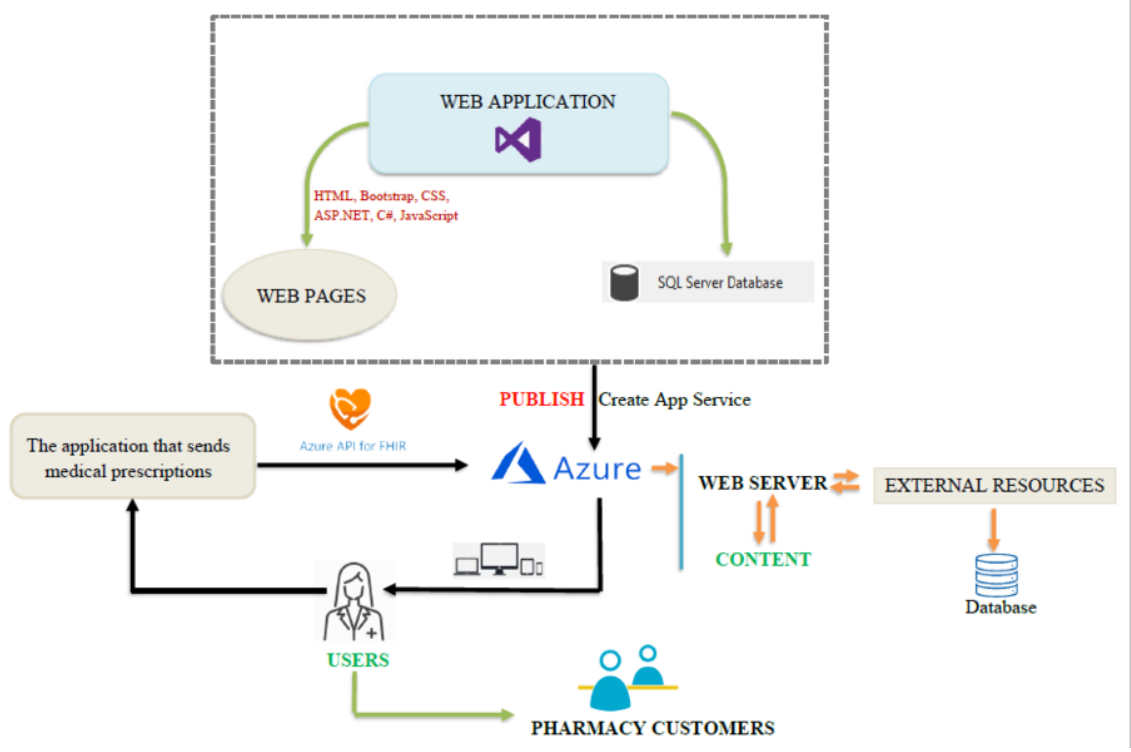

Figure 1. System architecture

The web-based application developed for pharmacies gives the possibility to receive prescriptions in the HL7 FHIR format. The web application supports prediction, and alerts if the medicine is not available in the pharmacy. It is developed using Visual Studio .NET 2017, using ASP.net pages and C\# language. The standardized communication uses the Microsoft Azure cloud facilities, with an API facilitating the communication in the standardized way.

\section{Conclusions}

It is important to have all the medical information in real time; this can be achieved using cloud computing and standardized communication. This paper presents initial work for supporting a medical unit to send a prescription using a standardized communication, with HL7 FHIR standards.

\section{References}

[1] IEEE Standard Glossary of Software Engineering Terminology. IEEE Std 610.12-1990.

[2] HL7 FHIR standard. Available from: https://www.hl7.org/fhir/. Accessed on 07.09.2021.

[3] Microsoft Azure. Available from: https://azure.microsoft.com/. Accessed on 06.09.2021.

[4] Pharmacy applications. Available from: https://softlead.ro/aplicatii-software/farmacii. Accessed on 08.09.2021. 